(C) [2009] IEEE. Reprinted, with permission, from Weng, Kaili., Nguyen, Tang., Nguyen, Hung., Su, Steven. Rate estimation for the monitoring of rehabilitation exercises. Proceedings of the 31st Annual International Conference of the IEEE Engineering in Medicine and Biology Society 2009. This material is posted here with permission of the IEEE. Such permission of the IEEE does not in any way imply IEEE endorsement of any of the University of Technology, Sydney's products or services. Internal or personal use of this material is permitted. However, permission to reprint/republish this material for advertising or promotional purposes or for creating new collective works for resale or redistribution must be obtained from the IEEE by writing to pubs-permissions@ieee.org. By choosing to view this document, you agree to all provisions of the copyright laws protecting it 


\title{
Rate estimation for the monitoring of rehabilitation exercises
}

\author{
Kaili WENG, Nghia Nguyen, Hung T.Nguyen, Senior Member, IEEE and Steven Su, Member, IEEE
}

\begin{abstract}
This study investigates the rate estimation problem encountered in rehabilitation exercise monitoring by using noninvasive portable sensors. The purpose of this paper has two main parts. The first part is to find suitable approaches for the rate detection of tri-axial accelerometer (TA) signals and ECG signals respectively. It is found that the integral type approaches (the short-time average magnitude difference function (AMDF) and short-term autocorrelation function (ACF)) are particularly suitable for $\mathrm{TA}$ signal pre-processing, while differential type approaches are very efficient for electrocardiographic (ECG) signal pre-processing. The second part is to develop a square wave matching method to detect the rate from the pre-processed signals. Experimental results indicate that the proposed methods can effectively detect pace rate from TA and heart rate from ECG and remove undesirable spikes.
\end{abstract}

\section{INTRODUCTION}

An increasing health problem in the developed world is that of chronic conditions associated with aging such as cardiovascular disease and diabetes. Exercise and regular daily physical activities are of vital importance in the management of chronic diseases [1,2]. In order to alleviate the burden of supervision placed on health professionals during the cardiovascular rehabilitation of a patient, wireless sensor based healthy monitoring systems are well developed. This paper investigates rate estimation approaches for the detection of exercises rate and heart rate from wireless tri-axial accelerometer and ECG signals respectively. Exercise rate is a direct reflection of exercise intensity. The heart rate is a measurement of cardiovascular response to exercise $[3,4]$. The analysis of heart rate has also proven useful in understanding cardiovascular regulation in a range of conditions, including heart failure, diabetes and hypertension $[5,6]$.

The Autocorrelation Function (ACF) and Average Magnitude Difference Function (AMDF) are widely used for rate detection [7]. As they involve integral (or summation) type calculations, we called them integral type approaches.

Kaili WENG is with Faculty of Engineering, University of Technology, Sydney, Broadway, NSW 2007, Australia (phone: +612-9514-2451; fax: +6 29514 2868; e-mail: kweng@eng.uts.edu.au).

Nghia Nguyen is with Faculty of Engineering, University of Technology, Sydney, Broadway, NSW 2007, Australia (phone: +612-9514-2451; fax: +61 29514 2868; e-mail: tnnguyen@eng.uts.edu.au)

Hung .T Nguyen is with Faculty of Engineering, University of Technology, Sydney, Broadway, NSW 2007, Australia (e-mail: Hung.Nguyen@uts.edu.au).

Steven $\mathrm{Su}$ is with Faculty of Engineering, University of Technology, Sydney, Broadway, NSW 2007, Australia (e-mail: Steven.Su@uts.edu.au).
These types of approaches are effective for random noise reduction. For the rate detection of tri-axial accelerometer (TA), integral type approaches have been built based on ACF and AMDF to eliminate random noise.

The short-term autocorrelation function (ACF) is one of the popular pitch detection methods, which can be written as (1):

$$
A C F(m)=\frac{1}{N-m+1} \sum_{n=m}^{N} x(n) x(n-m) . \quad 0 \leq \mathrm{m}<\mathrm{N}
$$

Where $x(n)$ are the samples of input discrete signal, $x(n-m)$ are the samples time shifted m periods, and $\mathrm{N}$ is the length of an analysis frame. In practice, the single ACF algorithm is competent in diminishing random noise in signal processing.

The average magnitude difference function (AMDF) is one of the useful algorithms in the field of speech processing. It has been widely used for detecting pitch or fundamental frequency [8]. The AMDF of a discrete signal $x(n)$ is defined by (2):

$$
A M D F(m)=\frac{1}{N-m+1} \sum_{n=m}^{N}|x(n)-x(n-m)| .0 \leq \mathrm{m}<\mathrm{N}
$$

Where $|x(n)-x(n-m)|$ denotes the absolute value of $x(n)-x(n-m)$.

AMDF and ACF are frequently used to analyze the fundamental frequency of a regular periodic signal. We applied these two methods in the preprocessing of TA signal. A real-time algorithm has been developed based on AMDF and ACF. We found that under strong random noise background, the combination of ACF and AMDF or repeat usage of ACF and AMDF are extremely effective $[9,10]$.

However, it is proven that the integral type approaches are not suitable for the pre-processing of ECG as they may reduce some high frequency signals, such as the $\mathrm{R}$ peak. Based on this consideration, the differential type function combined with a square wave matching detection algorithm has been developed (see Figure 1), which uses a square wave to match an ECG signal.

Even if ECG signals are not well represented by the device, heart rate can still be detected accurately by the square wave matching method. 


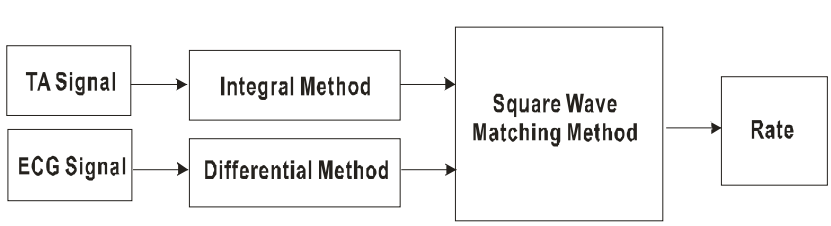

Figure 1. Block diagram of the proposed approach

The next section will describe the proposed rate detection approaches. In Section III, experimental results are given. Section IV concludes the paper.

\section{Methodology}

Methods for calculating rate based on ACF and AMDF have been introduced previously. The choice between them depends on the level of noise in the TA signal. For the white random noise, the ACF and AMDF can be used to detect the TA rate period. If the noise is strong, after ACF or AMDF are used once, the noise will be reduced, but the signal may still not be uniformly smooth. Therefore we can attempt to apply double $\mathrm{ACF}$, double AMDF or ACF+AMDF. These two integral methods will be compared and presented in the section III. Meanwhile, the differential method is optimal for the weak noise in ECG signals. The developed square wave matching approach is illustrated by using Figure 2 .

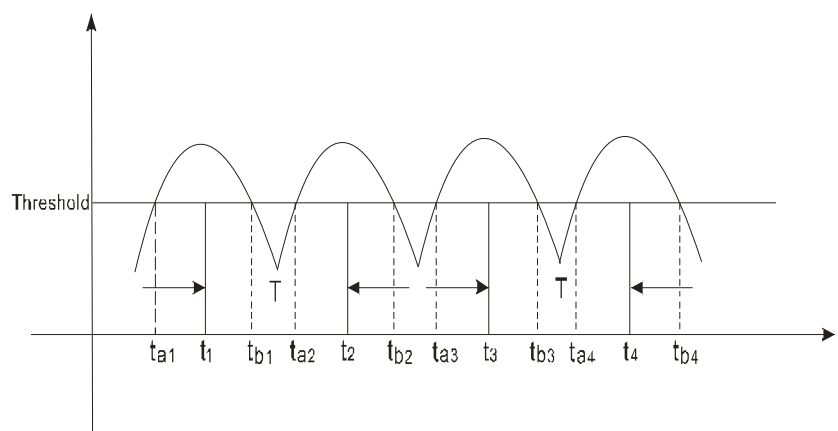

Figure 2. Description square wave matching approach

To apply the square wave matching method in the TA or heart rate signals, an appropriate threshold need to be selected. Assume the input signal is $x(k)$, then the generate square wave $y(k)$ can be described as:

$$
y(k)=\left\{\begin{array}{l}
1, \quad x(k) \geq \text { threshold } \\
0, \quad x(k) \leq \text { threshold }
\end{array}\right.
$$

The integers $t a i$ and $t b i$ can be estimated by using the following conditions:
For $t_{a i}$ and $t_{b i}, i=1,2,3 \ldots, k$ :

$$
\left\{\begin{array}{l}
y\left(t_{a i}\right)-y\left(t_{a i}-1\right)=1 \\
y\left(t_{b i}+1\right)-y\left(t_{b i}\right)=1
\end{array}\right.
$$

The next step is to calculate the $t_{i}$ by using the following equation:

$$
t_{i}=\frac{t_{a i}+t_{b i}}{2}
$$

The period $\mathrm{T}$ can be calculated as:

$$
T=\frac{1}{n} \sum_{i=1}^{n} t_{i}
$$

It is noticed that the estimated heart rate from using the proposed square wave matching method may be affected by the threshold values selected. In Figure 3, if the selected threshold value is $t h l$, we cannot detect the heart rate properly because there is no peak in the piece of ECG signals which can reach this threshold level. On the other hand, for the threshold th3, both P and T peaks will be counted. Therefore, the estimated rate should be divided by 2 . The threshold th 2 is a proper selection because only R peak can be detected by this threshold. To reduce threshold sensitivity, we pre-process the ECG signal by differentiation and obtained desired results (See Section III).

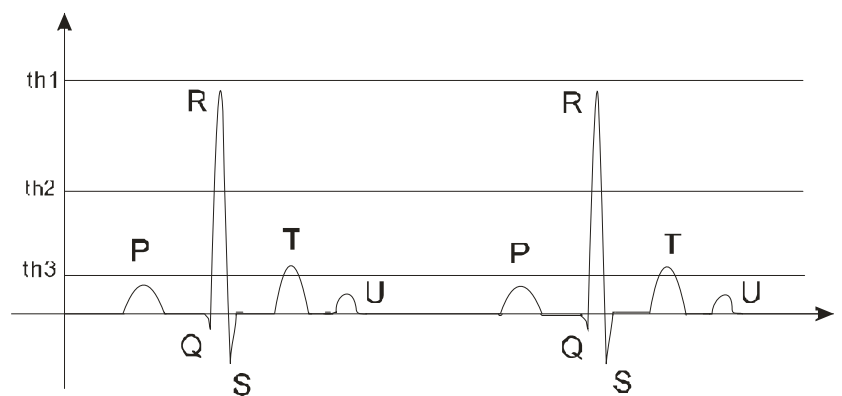

Figure 3. ECG signal with different thresholds.

\section{EXPERIMENTAL VERIFICATION}

In order to test the proposed approaches, we generated TA and ECG data by using "Heart and Activity Monitor"(HM131) manufactured by Alive Technologies Pty, Ltd Australia. The monitor has a built-in Bluetooth interface, which provides a wireless connection between the patient and host receiving system. 
ECG and accelerometer data is transmitted in real time over a Bluetooth SPP connection. The ECG signal was sampled at 300 samples per second, while the TA signal was sampled at 75 samples per second.

According to Equations (1) and (2), both ACF and AMDF have the advantage of being simple and effective for random noise reduction. The rate can be correctly detected from TA signal by using the integral type of approach (AMDF or ACF) (see Figure 4 and 5).

The $\mathrm{ACF}^{2}$ method, in contrast to the integral ACF or AMDF, has the advantage of making the TA signal smoother. Meanwhile, the ECG signal cannot be preprocessed by the integral method. Therefore, the differential and square wave matching methods are presented as shown in Figure 8.
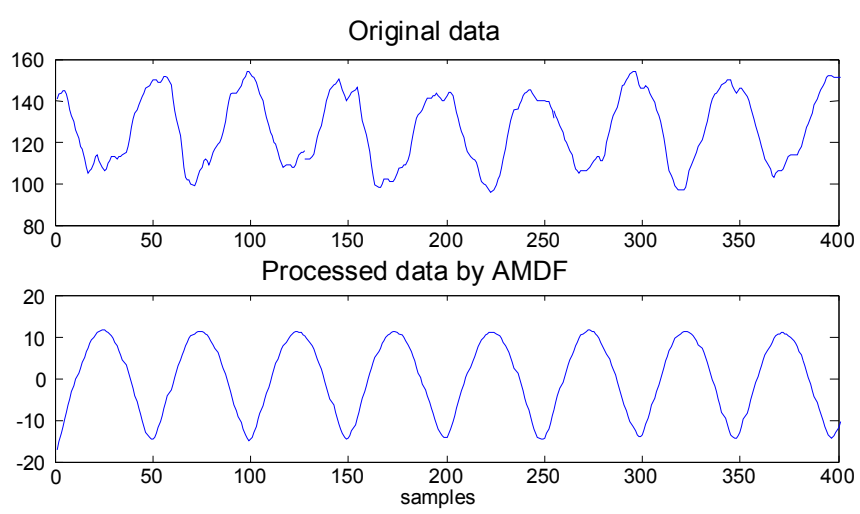

Figure 4. TA signal processing by AMDF
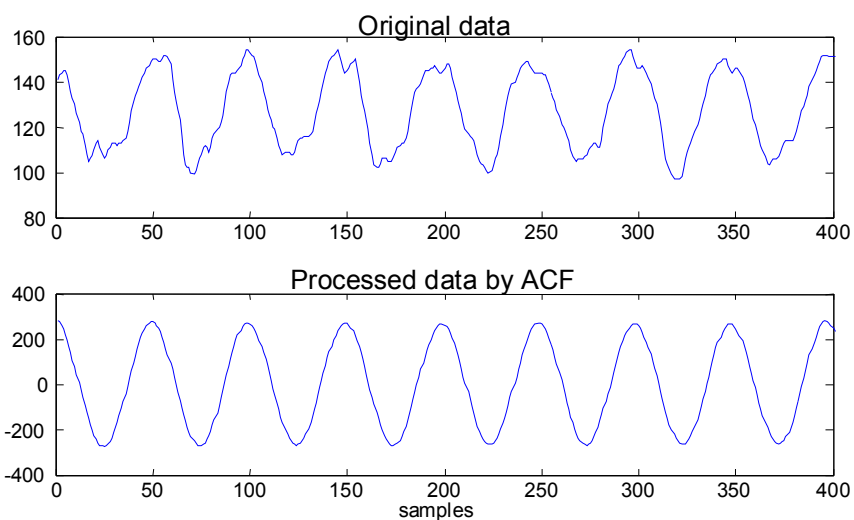

Figure 5. TA signal processing by using ACF

However, when noise is relatively high, the $\mathrm{AMDF}^{2}, \mathrm{ACF}^{2}$ or AMDF+ACF method should be applied (see Figure 6, 7 and 8).
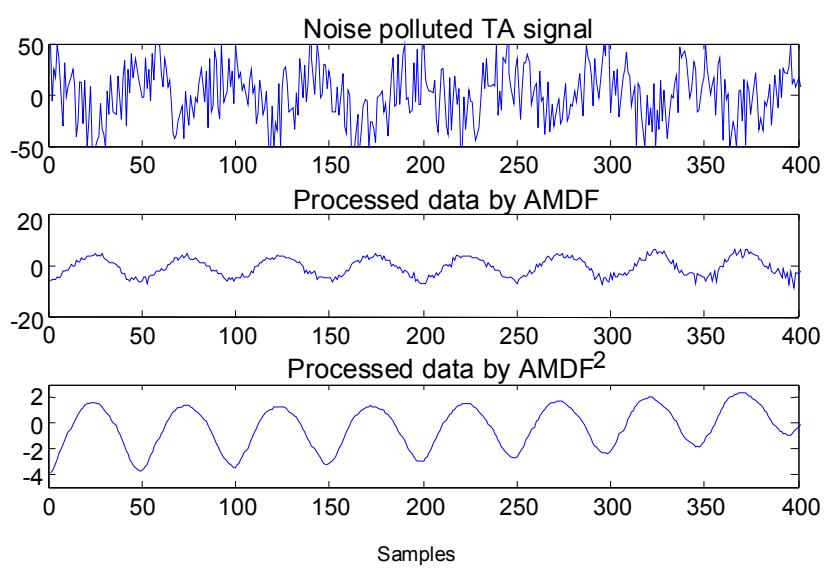

Figure 6. Noise polluted TA signal processing by using $\mathrm{AMDF}^{2}$
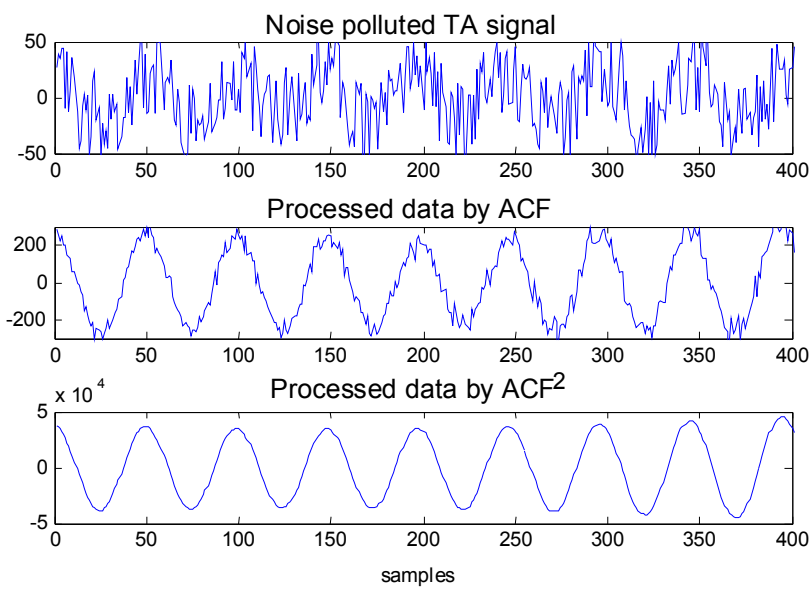

Figure 7. Noise polluted TA signal processing b y using $\mathrm{ACF}^{2}$
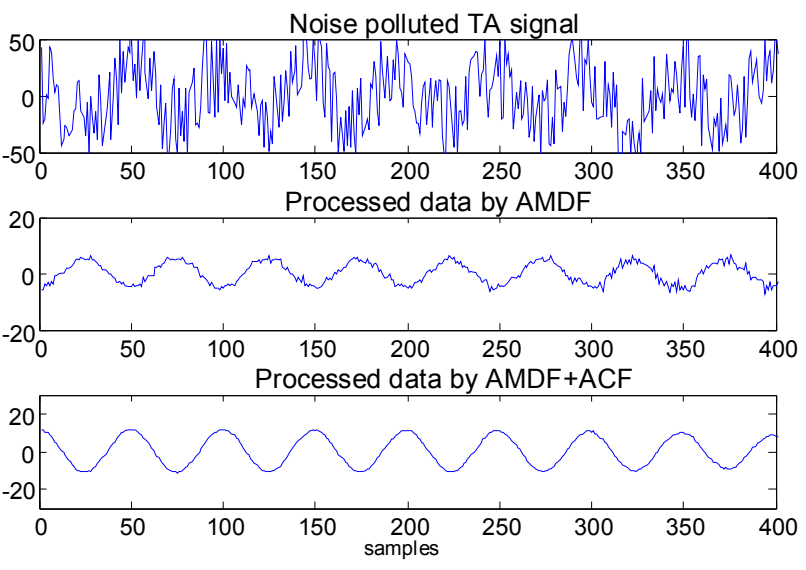

Figure 8. Noise polluted TA signal processing b y using AMDF+ACF 
As discussed before, the square wave matching method is sensitive to the selected threshold level. To minimize the sensitivity, the differential method has been implemented to pre-process the ECG signal. The proposed square wave matching method has been used to determine the heart rate from the preprocessed ECG signal as shown in Figure 9.
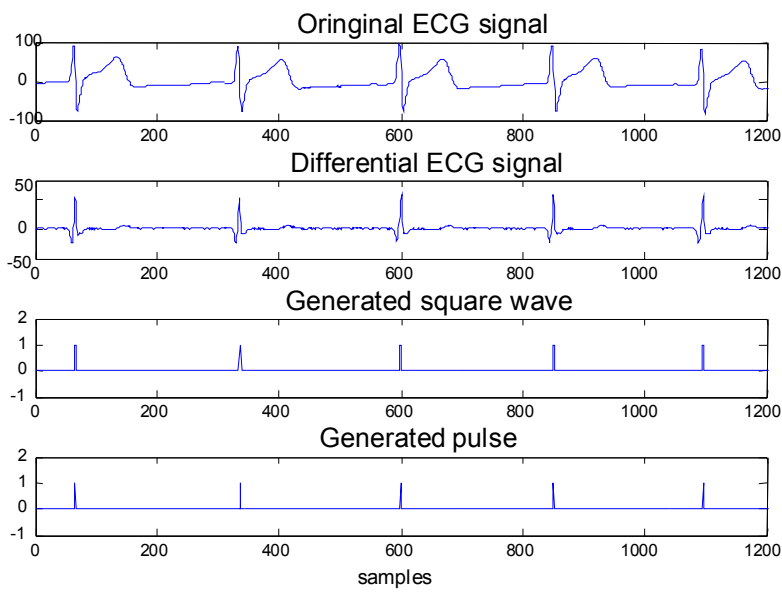

Figure 9. Heart rate detection

\section{CONCLUSION}

This paper mainly focuses on rate detection techniques for signals from noninvasive portable sensors. It was found that the repeat or combination usage of ACF and AMDF are very effective for TA signal processing. Experimental results have shown that repeat ACF obtains a better result in contrast with AMDF and simple ACF and their combination. Simulation and experiments also show that the differential type algorithm is suitable for ECG signal preprocessing. We developed a real time square wave matching method to detect the rate of preprocessed signals. In the next step, a more accurate square wave matching method based on threshold optimization and optimal filtering techniques will be developed.

\section{REFERENCE}

[1] Muhammad I. Ibrahimy, M.A. Mohd Ali and Edmond Zahedi, "An algorithm for the ambulatory monitoring of fetal heart rate," in proceedings of the $22^{\text {nd }}$ annual EMBS International conference Chicago July 23- 28, 2000 .

[2] Masakazu Asano, Yoshinori Tanabe, Keiko Watanabe, Hirokazu Genno, Ken-ichi Nemoto, Masako Isawa, Hiroshi Nose "Development of an exercise meter using triaxial acceleration data" in proceedings of the 2005 IEEE Engineering in Medicine and Biology $27^{\text {th }}$ annual conference Shanghai, China September 1-4, 2005

[3] Lambros V. Skarlas, Grigorios N. Beligiannis, Efstratios F.

Georgopoulos and Adam V. Adamopoulos "Intelligent modeling of the electrical activity of the human heart" in International Journal of Signal Processing1,1 winter 2005.

[4] Teddy M. Cheng, Andrey V. Savkin, Branko G. Celler, Steven W. Su, and Ning Wang, Universal algorithm for exercise rate estimation in walking, cycling, and rowing using triaxial accelerometry, Electronics Letters, 2009, will appear.

[5] Oscar Hernandez, Edgar olvera "Noise cancellation on ECG and heart rate signal using the undecimated wavelet transform in International" Conference on eHealth, pp. 145-150 Telemedicine and Social Medicine 2009 .

[6] Mingqi Chen, Olga Boric Lubecke, Victor Lubecke and Xiao yue Wang "analog signal processing for heart rate extraction" in proceedings of the 2005 IEEE Engineering in Medicine and Biology $27^{\text {th }}$ Annual Conference Shanghai, China, September 1-4, 2005

[7] Li Hui, Bei qian Dai and Lu wei "A pitch detection algorithm based on AMDF and ACF" in MOE-Microsoft Key Laboratory of Multimedia Computing and Communication, University of Science and Technology of China 2006.

[8] Hae Dong Kahng and Keun Sung Bae "Single channel adaptive doise cancelation for enhancing noisy speech" Internation Symposium on Speech, Image Processing and Neural Network, 13-16 April 1994 Hong Kong.

[9] L. Tan, and M. Karnjanadecha, "Pitch Detection Algorithm: Autocorrelation Method and AMDF," Proceedings of the 3rd International Symposium on Communications and Information Technology, Vol. II, pp. 541-546, Songkhla, Thailand, Sept., 3-5, 2003.

[10] Antti Vehkaoja, Jarmo Verho, Alper Comert, Baran Aydogan, Merja Perhonen, Jukka Lekkala, and Jouko Halttunen "System for ECG and heart rate monitoring during group training" in $30^{\text {th }}$ Annual International IEEE EMBS Conference Vancouver, British Columbia, Canada, August 20-24, 2008 